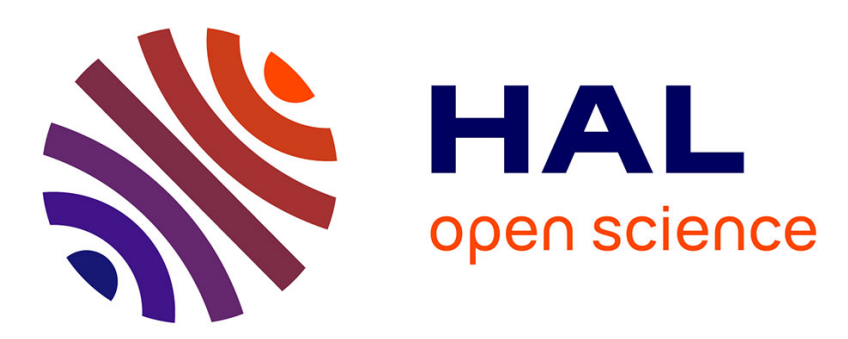

\title{
Modelization of the coupled behavior of a magnetically hard magnetorheological elastomer
}

Svenja Hermann, Christophe Espanet, Pauline Butaud, Gael Chevallier, Jean Manceau, Laurent Hirsinger

\section{- To cite this version:}

Svenja Hermann, Christophe Espanet, Pauline Butaud, Gael Chevallier, Jean Manceau, et al.. Modelization of the coupled behavior of a magnetically hard magnetorheological elastomer. 11th European Conference on Constitutive Models for Rubber, Jun 2019, Nantes, France. hal-02370740

\author{
HAL Id: hal-02370740 \\ https://hal.science/hal-02370740
}

Submitted on 19 Nov 2019

HAL is a multi-disciplinary open access archive for the deposit and dissemination of scientific research documents, whether they are published or not. The documents may come from teaching and research institutions in France or abroad, or from public or private research centers.
L'archive ouverte pluridisciplinaire HAL, est destinée au dépôt et à la diffusion de documents scientifiques de niveau recherche, publiés ou non, émanant des établissements d'enseignement et de recherche français ou étrangers, des laboratoires publics ou privés. 


\title{
Modelization of the coupled behavior of a magnetically hard magnetorheological elastomer
}

\author{
S. Hermann \& C. Espanet \\ Research and Development Department, Moving Magnet Technologies SA, Besançon, France. \\ P. Butaud, G. Chevallier, J.F. Manceau \& L. Hirsinger \\ Univ. Bourgogne Franche-Comté FEMTO-ST, CNRS/UFC/ENSMM/UTBM, Besançon, France.
}

\begin{abstract}
The key issue of this contribution is the magneto-mechanical behavior of a magnetically hard magnetorheological elastomer (H-MRE). A numerical model based on the conservation laws of continuum mechanics and magnetism is presented and then applied to a specific 2-dimensional case of an H-MRE specimen. The interaction between two remanently magnetized particles and its dependence on the relative positioning of the particles to one another are studied. The reaction of the elastomer matrix to the particle-particle interaction is analyzed for three configurations of the particles in the specimen. In a second step, the specimen is exposed to a unidirectional tensile load. It is demonstrated that the magnetization of the particles induces a change in the modulus which strongly depends on the arrangement of the particles in the matrix: an increase as well as a decrease of the modulus are observed. By the help of the principal stresses, three different types of magnetic interactions are identified and assigned to the corresponding softening and stiffening reactions of the specimen.
\end{abstract}

\section{INTRODUCTION}

The term magnetorheological elastomer (MRE) describes a composite material which consists of magnetosensitive particles dispersed in an elastomer matrix. MRE are interesting for engineering applications because their mechanical properties can be influenced by magnetic fields. The particles can be either dispersed randomly in the matrix (isotropic MRE) or be aligned in chains (anisotropic MRE). To align the particles, the composite is cured in an external magnetic field (Rigbi and Jilkén 1983, Ginder et al. 1999). MRE can furthermore be divided in S-MRE, containing magnetically soft particles and H-MRE in which magnetically hard particles like $\mathrm{NdFeB}$ are present.

\subsection{Modelization of MRE}

In order to better understand the magnetomechanical behavior of S-MRE observed in experiments, several models have been developed over the last years as the review of Cantera et al. (2017) shows. A coupled model can be based on the conservation laws of continuum mechanics and magnetism as well as on the potential energy (Danas et al. 2016). In both cases, an external magnetic field is necessary to generate magnetic interactions in an S-MRE as the particles cannot be remanently magnetized. The particles are of- ten considered as spherical and their magnetization as unidirectional, as for example in the work of Jolly et al. (1996). By analyzing the magnetic interaction energy between aligned particles, a raise of the shear stiffness due to the magnetic field can be found. In a parametrical study conducted by Han et al. (2013), straight chains are compared to wavy particle chains. It is shown that the modulus varies with the particles' misalignment and an increase as well as a decrease is observed. An analytical model for randomly distributed particles has been proposed by Borcea and Bruno (2001). They study the macroscopic behavior of an S-MRE with a statistically homogeneous distribution of particles. A study of the magnetic forces between two spherical, magnetically soft particles is presented as well.

The work presented in the following is using the direct method to model the behavior of MRE. In contrast to most of the models existing in the literature, the numerical model presented in the following concerns an H-MRE. Instead of an external magnetic field creating magnetic dipoles in the matrix, the remanently magnetized particles generate a magnetic field. The model is implemented in the finite element software COMSOL and the magnetic interactions between two magnetic dipoles as well as their impact on the elastomer matrix are studied. 


\section{MAGNETOMECHANICAL MODEL}

\section{$2.1 \quad$ Fundamental assumptions}

Several assumptions are made for the numerical model, on the one hand to simplify the validation of the model and on the other hand to reduce calculation time. The influence of time depending effects is neglected for both physical domains which means that creep or relaxation behavior is not considered. A static study without electric current is assumed. A rigid connection between the particles and the matrix is supposed. Figure 1 sketches the general case of a magnetic particle $\Omega_{p}$ enclosed by an elastomer matrix $\Omega_{e}$ in a volume of air $\Omega_{a}$. The interfaces between two materials or boundaries are denoted with the symbol $\Gamma$. In the following section the magnetomechanical model is presented on the example of this general case.

\subsection{Governing equations}

The absence of currents allows the definition of a magnetic scalar potential $V_{m}$ which is related to the magnetic field $\vec{H}$ by equation (1). The magnetic behavior is assumed to be linear for all materials. In this case, the relation between the magnetic field and the magnetic flux density $\vec{B}$ is established by the vacuum permeability $\mu_{0}$ and the relative permeability $\mu_{r}$ of a material. A remanent magnetic flux density $\vec{B}_{r}$ is associated with the magnetized material while for the air and the elastomer $\vec{B}_{r}$ is equal to the zero vector in equation (2). The magnetic flux is conserved by the Maxwell equation (3). The magnetic stress tensor $\sigma^{m a g}$ is obtained from the magnetic field, the magnetic flux density and the magnetization $\vec{M}$ of the different materials (4).

The mechanical material behavior is assumed to be linear. For an isotropic linear elastic behavior, the deformation tensor $\varepsilon$ is linked to the mechanical stress tensor $\boldsymbol{\sigma}^{\text {mech }}$ by the fourth order elasticity tensor $C(5)$ which can be obtained from the Young modulus $E$ and the Poisson's ratio $\nu$. The deformation can furthermore be expressed as a function of the displacement field $\vec{u}(6)$.

In a static case and under absence of external volume forces the magnetomechanical coupling can be established in different ways. In a volume based approach, equation (7) describes the fact that the magnetic and mechanical stresses counterbalance each other in the equilibrium state. The coupling can also be established by a boundary condition since the stress state has to be continuous on a boundary between two materials. According to Eringen and Maugin (1990), the boundary condition at the interface between the particle and the elastomer can be expressed with equation (8) for a quasi-static case. $\llbracket X \rrbracket=X^{+}-$ $X^{-}$corresponds to the tensor jump at the interface, the normal vector $\vec{n}_{p}$ is pointing from - to + . The indices $p$ and $e$ of the mechanical and the magnetic stress tensors, $\boldsymbol{\sigma}^{\text {mech }}$ and $\boldsymbol{\sigma}^{\text {mag }}$, stand for the particle and the elastomer domain.

When post-processing the results of the magnetomechanical problem, an equivalent magnetic force $\vec{F}$ acting on the particle's center of gravity $G$ can be calculated by the help of $\boldsymbol{\sigma}^{\text {mag }}$ from equation (9). In the same way, an equivalent torque $\vec{T}$ with respect to $G$ is introduced by summing the contribution of each point $C$ of the boundary $\Gamma_{e p}$ (10) (COMSOL 2019).

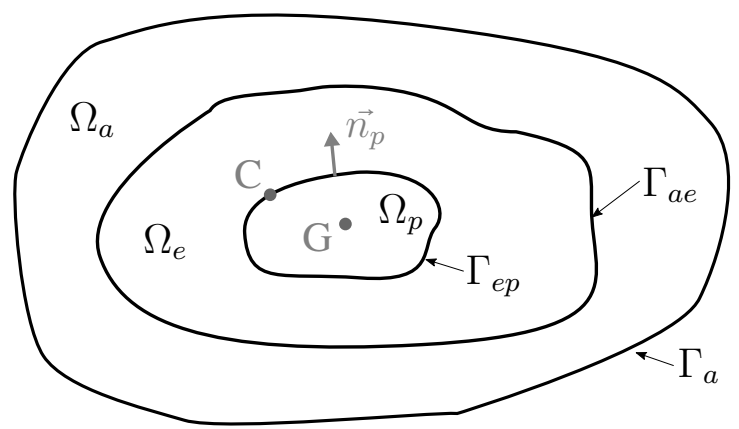

Figure 1: Sketch of a magnetic particle $\Omega_{p}$ surrounded by a elastomer matrix $\Omega_{e}$ in a volume of air $\Omega_{a}$.

$$
\begin{array}{lr}
\vec{H}=-\operatorname{grad}\left(V_{m}\right) & \text { in } \Omega_{a} \cup \Omega_{e} \cup \Omega_{p} \\
\vec{B}=\mu_{0} \mu_{r} \vec{H}+\vec{B}_{r} & \text { in } \Omega_{a} \cup \Omega_{e} \cup \Omega_{p} \\
\operatorname{div}(\vec{B})=0 & \text { in } \Omega_{a} \cup \Omega_{e} \cup \Omega_{p} \\
\boldsymbol{\sigma}^{m a g}=-\frac{1}{2 \mu_{0}}(\vec{B} \cdot \vec{B}) \boldsymbol{I}+(\vec{M} \cdot \vec{B}) \boldsymbol{I} \\
+\frac{1}{\mu_{0}} \vec{B} \otimes \vec{B}-\vec{M} \otimes \vec{M} & \text { in } \Omega_{e} \cup \Omega_{p}
\end{array}
$$

$$
\begin{array}{ll}
\boldsymbol{\sigma}^{\text {mech }}=\boldsymbol{C}: \boldsymbol{\varepsilon} & \text { in } \Omega_{e} \cup \Omega_{p} \\
\boldsymbol{\varepsilon}=\frac{1}{2}\left(\operatorname{grad}(\vec{u})+\operatorname{grad}(\vec{u})^{T}\right) & \text { in } \Omega_{e} \cup \Omega_{p}
\end{array}
$$

$$
\begin{aligned}
& \operatorname{div}\left(\boldsymbol{\sigma}^{\boldsymbol{m a g}}+\boldsymbol{\sigma}^{\text {mech }}\right)=\overrightarrow{0} \quad \text { in } \Omega_{e} \cup \Omega_{p} \\
& \vec{n}_{p}^{T} \llbracket \boldsymbol{\sigma}^{m a g}+\boldsymbol{\sigma}^{\text {mech }} \rrbracket=\vec{n}_{p}^{T}\left(\boldsymbol{\sigma}_{\boldsymbol{e}}^{\text {mag }}+\boldsymbol{\sigma}_{\boldsymbol{e}}^{\text {mech }}\right. \\
& \left.-\sigma_{p}^{m a g}-\sigma_{p}^{m e c h}\right)=\overrightarrow{0} \\
& \text { on } \Gamma_{e p}
\end{aligned}
$$

$\vec{F}=\oint_{\Gamma_{e p}}\left({\overrightarrow{n_{p}}}^{T} \boldsymbol{\sigma}_{e}^{m a g}\right) d A$

$$
\vec{T}=\oint_{\Gamma_{e p}}\left(\overrightarrow{G C} \times\left(\vec{n}_{p}^{T} \boldsymbol{\sigma}_{e}^{m a g}\right)\right) d A
$$




\subsection{Application to a specific case}

A 2D magneto-mechanical case (Figure 2) has been set up in COMSOL 5.3a. The problem is reduced to 2 dimensions by assuming a mechanical plane stress behavior and forcing the magnetic field to be plane as well. The $\mathrm{x}-\mathrm{y}$ plane is chosen as working plane while the out-of-plane thickness, corresponding to the thickness in the $\mathrm{z}$-direction, is set to $t=1 \mu \mathrm{m}$. The model comprises a micron sized H-MRE specimen located in a volume of air $\Omega_{a}$ with the side length of $L_{a}=100 \mu \mathrm{m}$. Two magnetic particles, $\Omega_{p_{1}}$ and $\Omega_{p_{2}}$, with a circular cross section and a diameter of $d=5 \mu \mathrm{m}$ are enclosed by an elastomer matrix $\Omega_{e}$ having a side length of $L_{e}=25 \mu \mathrm{m}$. A vector $\vec{e}$ is starting from the center of $\Omega_{p_{1}}$, passing by the point $O(x=0 \mid y=0)$ and pointing towards the center of $\Omega_{p_{2}}$. The distance between the particles is described by the length of the vector $\vec{e}$.

In a parametric study the angular position of the particles to one another is altered. The distance $|\vec{e}|$ is kept constant at $11 \mu \mathrm{m}$ while the angle $\theta$ between $\vec{e}$ and the direction of the particles' magnetization (coincident with the y-direction) changes. The range for $\theta$ is set from $0^{\circ}$ to $180^{\circ}$ and divided into steps of $5^{\circ}$. With the given range for $\theta, 37$ configurations are obtained.

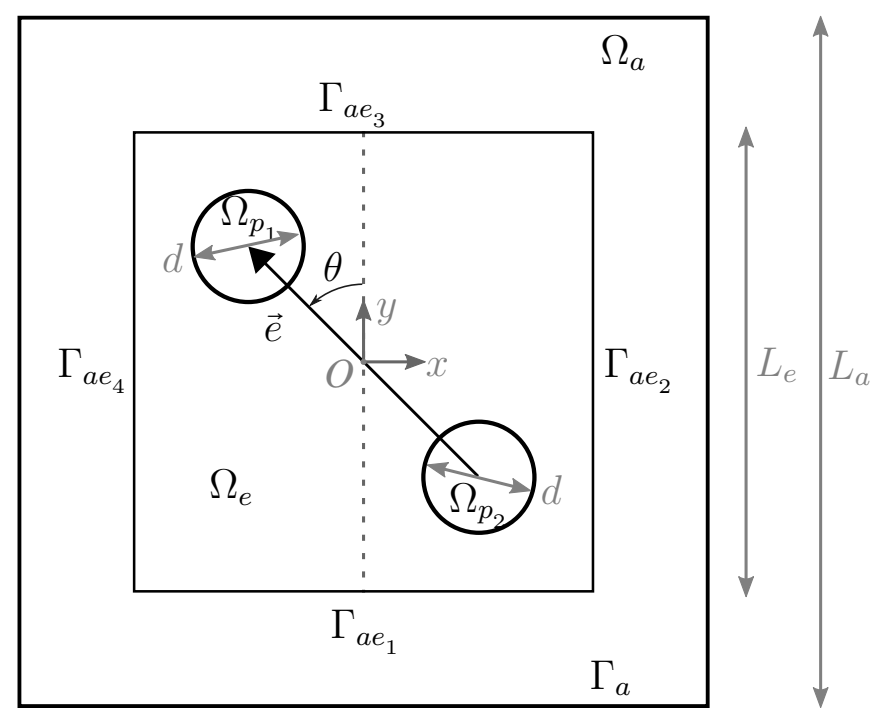

Figure 2: Sketch of specific case of application for the model.

All properties of the different materials used for the specific case are summarized in Table 1 . The relative magnetic permeability $\mu_{r}$ is set to 1 for the air and the elastomer. Due to the experience in magnetic simulation of the company MMT, $\mu_{r}$ is set to 1.3 for the magnetic material NdFeB. The elastomer is considered as a nearly incompressible material, its Poisson ratio $\nu$ is set to 0.499 . The Young modulus $E$ of $0.7 \mathrm{MPa}$ is obtained from a quasi-static tensile test on an elastomer of which the density $\rho=1.08 \mathrm{~g} / \mathrm{cm}^{3}$ is given in the datasheet. The company Neorem Magnets OY (2019) discloses a value of $150 \mathrm{GPa}$ for the Young modulus of sintered $\mathrm{NdFeB}$ magnets which is used for the magnetic material in this case. The Poisson ratio is set to
0.3. A y-component for the remanent magnetization of $B_{r_{y}}=0.8 \mathrm{~T}$ is associated with the two particles. This magnitude as well as a density of $7.62 \mathrm{~g} / \mathrm{cm}^{3}$ are given in the datasheet of the magnetic powder MQFP14-12-20000-089 from Magnequench.

Table 1: Material properties

\begin{tabular}{|c|c|c|c|c|c|}
\hline & \multicolumn{2}{|c|}{ magnetic } & \multicolumn{2}{|c|}{ mechanical } & \multirow[b]{2}{*}{$\rho\left[\mathrm{g} / \mathrm{cm}^{3}\right]$} \\
\hline & $\mu_{r}$ & $\vec{B}_{r}[\mathrm{~T}]$ & $E[\mathrm{MPa}]$ & $\nu$ & \\
\hline$\Omega_{a}$ & 1 & $\left(\begin{array}{ll}0 & 0\end{array}\right)^{T}$ & - & - & - \\
\hline$\Omega_{e}$ & 1 & $\left(\begin{array}{ll}0 & 0\end{array}\right)^{T}$ & 0.7 & 0.499 & 1.08 \\
\hline$\Omega_{p}$ & 1.3 & $\left(\begin{array}{lll}0 & 0.8\end{array}\right)^{T}$ & 150000 & 0.3 & 7.62 \\
\hline
\end{tabular}

Regarding the mesh, a quadratic interpolation is chosen for the magnetic scalar potential as well as for the displacement field. The deformation of the composite is taken into account for the calculation of the magnetic forces.

\section{PARAMETRIC STUDY}

\subsection{Particle-particle interaction}

To study the interaction between the magnetized particles and the resulting stress state in the matrix, the boundaries of the elastomer are fixed. The magnetic flux can propagate in the material but not leave the air domain. The boundary conditions can be written as

$$
\begin{array}{ll}
\vec{u}=\overrightarrow{0} & \text { on } \Gamma_{a e_{1}}, \Gamma_{a e_{2}}, \Gamma_{a e_{3}}, \Gamma_{a e_{4}}, \\
\vec{n}_{a}^{T} \vec{B}=0 & \text { on } \Gamma_{a} .
\end{array}
$$

The evolution of the magnetic force components, $F_{x}$ and $F_{y}$, as well as the torque around the z-axis $T_{z}$ are calculated for each particle with respect to its center. Figure 3 shows the results obtained for $\Omega_{p_{1}}, \Omega_{p_{2}}$ and $T_{z}$ as function of $\theta$. The evolution of all the force components with $\theta$ is sinusoidal and shows a periodicity of $120^{\circ} . F_{x}$ has the same magnitude for $\Omega_{p_{1}}$ and $\Omega_{p_{2}}$ but the components are oppositely directed. The same result is obtained for $F_{y}$. The configurations for which $F_{x}$ reaches a maximum are the configurations where $F_{y}$ is equal to zero and vice versa. The evolution of $T_{z}$ with $\theta$ is sinusoidal and shows a periodicity of $180^{\circ}$.

The result for $\theta=0^{\circ}$ is comprehensible: the particles are aligned in the $y$-direction, different magnetic poles face each other and attraction forces in the y-direction appear. Since the particles are perfectly aligned, the force does not contain a component in the $\mathrm{x}$-direction. The torque is zero because the particles do not need to rotate to align their magnetic moments as the alignment is given by their arrangement. The remaining results are more difficult to understand without a visualization. Therefore, 9 configurations for which either the force or the torque reach an extreme value or are equal to zero are sketched in Figure 4. 
The particles and their magnetic moment (black arrow) are shown as well as the force vector (straight red arrow) and a curved arrow representing the torque. As described before, an alignment of the particles in the y-direction, corresponding to $\theta=0^{\circ}$ and $\theta=180^{\circ}$ results in two opposing magnetic poles facing each other (Figure 4a and 4i). To reduce the magnetic energy they tend to form one dipole from the two existing ones and the particles align. For $\theta=90^{\circ}$, two north poles and two south poles face each other in the $\mathrm{x}$-direction (Figure 4e). Consequently the particles repel each other in this direction. There is no torque since in this perfectly symmetric configuration the stresses leading to a rotation compensate one another. An alignment of particles in the direction of their magnetization or perpendicular to it results in a force that causes a translation of the particle.
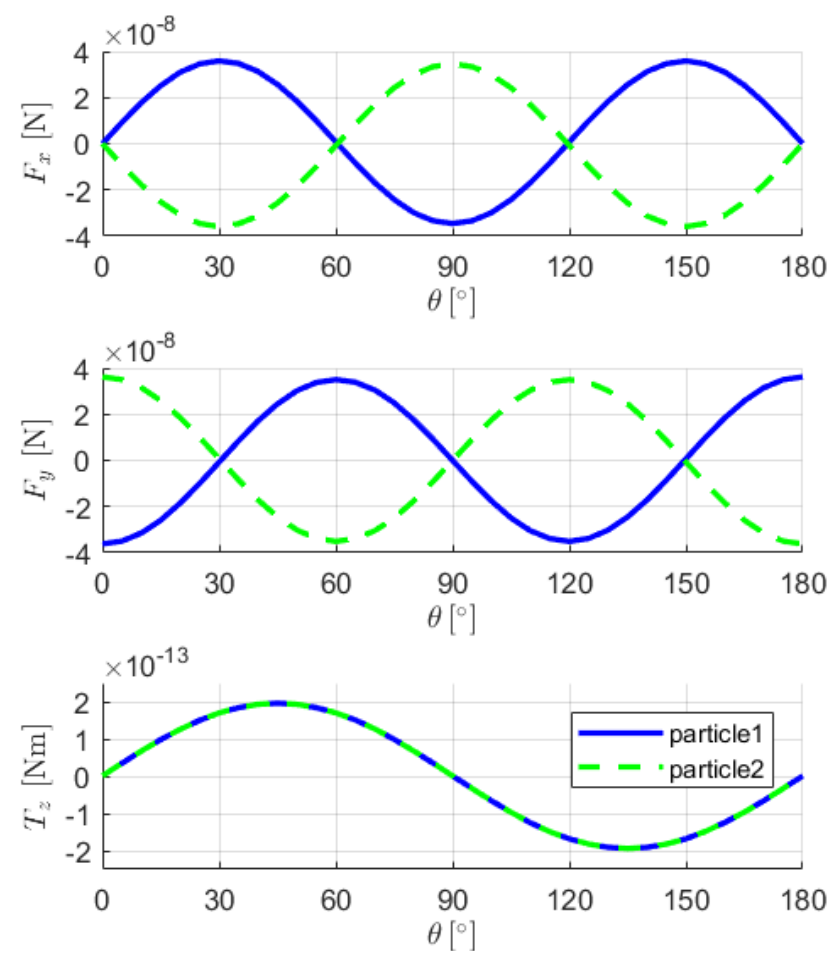

Figure 3: Evolution of the components of the magnetic force $F_{x}$, $F_{y}$ and the torque $T_{z}$ with the angle $\theta$

The remaining six configurations are intermediate states for which a torque is acting on the particles additionally to the force. A maximum torque is reached for $\theta=45^{\circ}$ and $\theta=135^{\circ}$ (Figure $4 \mathrm{c}$ and g). It can also be seen that the forces for 4 configurations, namely the ones with $\theta=30^{\circ}, 60^{\circ}, 120^{\circ}$ and $150^{\circ}$, are unidirectional (Figure $4 \mathrm{~b}, 4 \mathrm{~d}$, 4f and $4 \mathrm{~h}$ ). But in contrast to the configurations with aligned particles in $\mathrm{x}$ - or $\mathrm{y}$-direction, a torque is acting on the particles. The reason for this are the Maxwell stresses. Since the force is a global value, calculated as an integral over $\Gamma_{e p}$ (equation (10)), opposing stresses are canceled out by the summation. In the case of $\theta=30^{\circ}$ for example, the normal stresses in the y-direction compensate each other. Nevertheless the tangential components are present and cause a torque.

As a consequence of the particle-particle interaction, the elastomer is exposed to mechanical stresses.
The principal stresses are analyzed for three configurations, for which $\theta$ takes a value of $0^{\circ}, 45^{\circ}$ and $90^{\circ}$ respectively (Figure 5 ). The arrows indicate the direction of the stress and the color is representative for the magnitude. A negative value corresponds to a compressive stress and a positive value to a tensile stress.

As it can be seen in Figure 5a, the elastomer between the particles is under compressive stress while the material near the upper and lower boundary is under tensilte stress for $\theta=0^{\circ}$. For an angle of $\theta=$ $90^{\circ}$, the local stress behavior is the exact opposite: the elastomer between the particles is under tensile stress while compressive stress appears in the zone near the lateral boundaries. For the configuration corresponding to $\theta=45^{\circ}$, the zone between the particles shows tensile and compressive stresses in the principal directions. By the help of a coordinate transformation it could be shown that the state is equivalent to pure shear stress. At the same time, the matrix at the lateral boundaries of the elastomer is under tensile stress while the material near the upper and lower boundaries is under compressive stress. The magnetic torque and the tendency of the particles to align in the direction of magnetization is the origin of a complex local stress behavior in the elastomer.
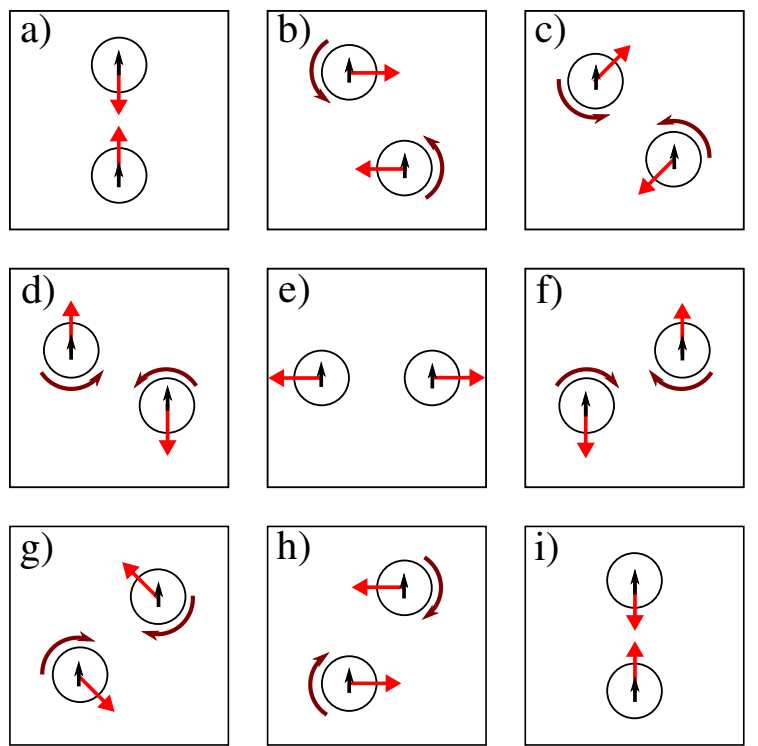

Figure 4: Arrangement of the particles, magnetic moment (black arrow), magnetic force (straight red arrow) and torque (curved red arrow) for 9 configurations of the parametric study.

\subsection{Macroscopic behavior}

In a next step, the influence of the particles' position on the macroscopic behavior of the specimen is evaluated: the equivalent of a unidirectional tensile test is simulated. Therefore a static displacement of $a=$ $L_{s} \cdot 1 \%$ is imposed to the specimen in the y-direction. The resulting deformation of the specimen is sketched in Figure 6a. While the magnetic boundary conditions stay the same as stated in equation (12), the mechanical boundary conditions for this case are the follow- 
a)

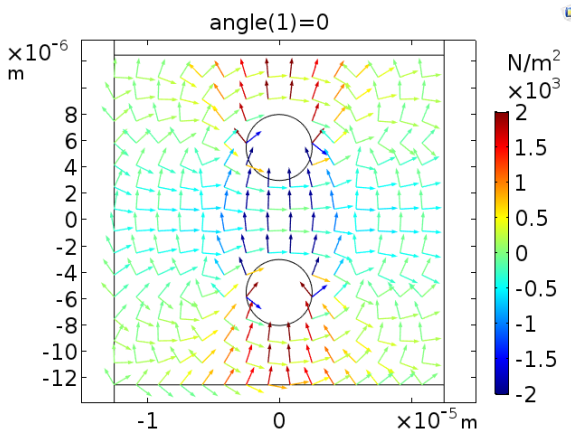

b)

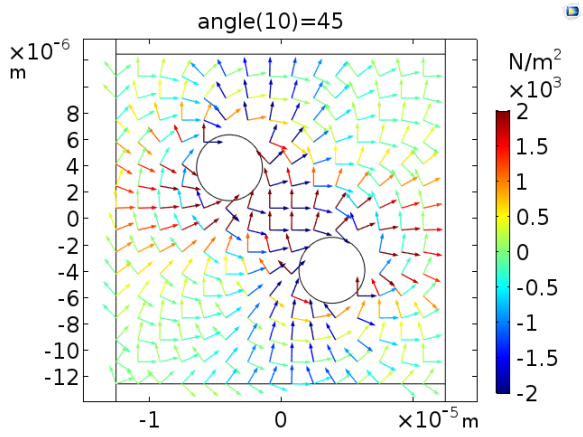

c)

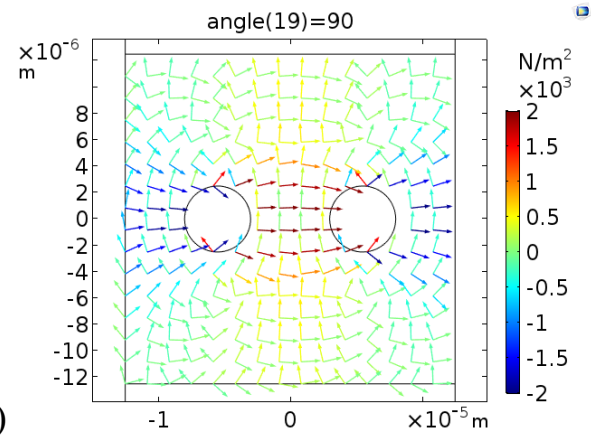

Figure 5: Principal stresses in the elastomer for the configurations $1\left(\theta=0^{\circ}\right), 10\left(\theta=45^{\circ}\right)$ and $19\left(\theta=90^{\circ}\right)$

ing:

$$
\begin{array}{ll}
\left|u_{y}\right|=0.5 \% \cdot L_{e} & \text { on } \Gamma_{a e_{1}}, \Gamma_{a e_{3}}, \\
\vec{u}=\overrightarrow{0} & \text { on } O .
\end{array}
$$

The apparent Young modulus of the specimen in this direction is obtained by dividing the y-component of the reaction force $\mathrm{Fr}_{y}$ on $\Gamma_{a e_{1}}$ by the theoretical cross section in the $\mathrm{x}-\mathrm{z}$ plane $\left(L_{e} \cdot t\right)$ and the elongation of $1 \%$. To study the influence of the magnetization on the apparent Young modulus, the test is conducted for the same configuration with nonmagnetized particles $\left(B_{r y}=0 T\right)$ and with magnetized particles $\left(B_{r y}=0.8 T\right)$. The ratio of the corresponding moduli $E\left(B_{r y}=0.8 T\right)$ and $E\left(B_{r y}=0 T\right)$ gives the change in the apparent Young modulus $\Delta E$ due to magnetization:

$$
\Delta E=\frac{E\left(B_{r}=0.8 T\right)}{E\left(B_{r}=0 T\right)} .
$$

The evolution of $\Delta E$ as a function of the angle $\theta$ is shown in Figure 6b. In three zones, the ratio is higher than 1 which corresponds to a stiffening of the material due to the magnetization of the particles. In the two zones where the ratio is smaller than 1, the magnetic interaction of the particles leads to a material softening. The magnetic forces and principal strains in the matrix can be helpful to understand that there are three different effects leading to the change in the modulus.

The first one is the stiffening appearing for an angle $\theta$ from $0^{\circ}$ to approximately $24^{\circ}$ and $154^{\circ}$ to approximately $180^{\circ}$. The corresponding ranges are labeled as 'effect 1 ' in Figure $6 \mathrm{~b}$. The example of configuration 1 with $\theta=0^{\circ}$ is chosen to explain the effect. The particles attract each other and so the magnetic forces are opposing the mechanical forces. As a perfect cohesion between particles and matrix is supposed, tensile stresses appear on the matrix upper and lower bounds (Figure 5a). A higher magnitude of force is necessary to reach an elongation of the specimen, the modulus increases as a consequence.

The apparent stiffening in the zone where $\theta$ ranges from approximately $72^{\circ}$ to $108^{\circ}$ is caused by the incompressibility of the matrix. For $\theta=90^{\circ}$ there are repelling forces between the particles in the $\mathrm{x}$-direction.
Since the distance between the particles increases in this direction, there are compressive stresses appearing in the $\mathrm{x}$-direction (Figure 5c). Due to the incompressibility of the matrix, as it tends to elongate in the $\mathrm{x}$-direction it shrinks in the y-direction. The stress state is opposite to the one imposed by the tensile test and this causes the modulus to increase. The corresponding range is labeled with 'effect 3' Figure $6 b$.

The attempt of the particles to align in the ydirection is the reason for the material softening in the zone labeled as 'effect 2' in Figure 6b. As it can be seen for the principal stress state of $\theta=45^{\circ}$, the particle interaction causes a tensile stress on the lateral boundaries of the elastomer and compressive stress on the upper and lower boundary. These stresses contribute to a traction in the y-direction and less force is necessary to elongate the specimen, as a consequence the modulus decreases.

It is important to mention that there are three configurations for which the magnetism has no influence on the modulus $(\Delta E=1)$. The mean of $\Delta E$ is also equal to 1 which means that the softening and stiffening effects are likely to compensate each other statistically in a bigger specimen with randomly distributed particles.

\section{CONCLUSIONS}

\subsection{Summary of the results}

In this work, a magnetomechanical model for an $\mathrm{H}-\mathrm{MRE}$ based on the conservation laws of continuum mechanics and magnetism is presented. After a brief contextual setting of the research field, a summary of the general assumptions is given and the governing equations are presented. The model is applied to the simple case of two interacting hard magnetic particles embedded in an elastomer.

A specimen consisting of a elastomer matrix which is surrounded by air while containing two remanently magnetized particles is used to study the influence of the microstructure on the magnetomechanical behavior of an H-MRE specimen. The angular distance between the particles has been altered from $0^{\circ}$ to $180^{\circ}$ by steps of $5^{\circ}$. For the 37 resulting configurations the magnetic forces and the torque as well as the impact 


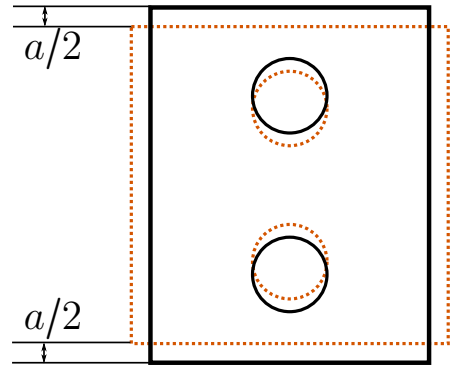

a)

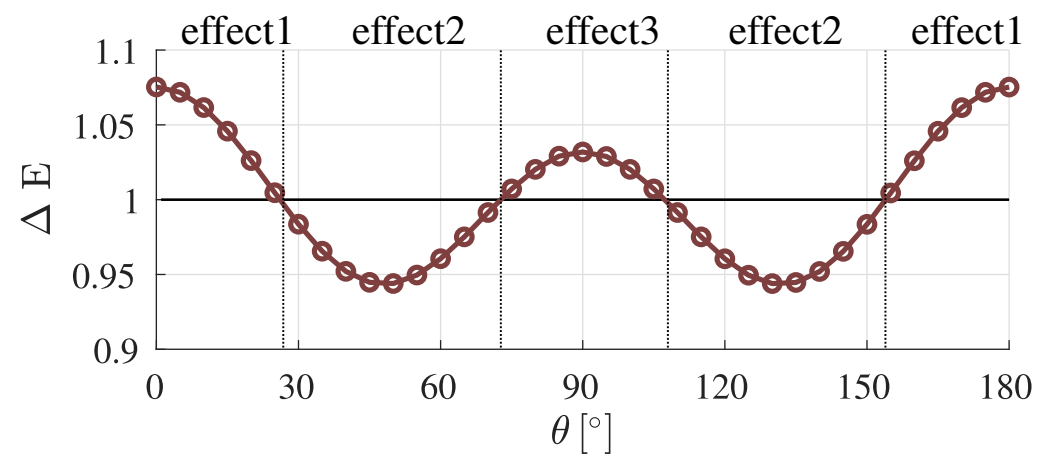

Figure 6: (a) Scheme of the specimen in original and elongated state (orange and black line respectively) and (b) evolution of $\Delta E$ as a function of the angle $\theta$ including the identification of five zones in which three different effects lead either to a material stiffening or softening

on the stress state of the elastomer matrix have been evaluated. While forces acting on the particles show a periodicity of $120^{\circ}$, the torque is $180^{\circ}$ periodic. It has been shown that the forces as well as the torque are important to understand the stress arising in the elastomer matrix. The orientation of the magnetic forces corresponds to the results for the analytical model of Borcea \& Bruno (2001), but in contrast to their model developed for an S-MRE, a torque is present due to the magnetic anisotropy induced by the magnetization of the particles.

In a next step, the influence of the microstructure in combination with the remanent magnetization of the particles has been studied. Uniaxial tension has been applied to the specimen in its 37 configuration. The apparent Young modulus of a non-magnetized specimen and a magnetized specimen of same configuration are compared. It can be stated that the microstructure has an important influence on the macroscopic behavior since this apparent modulus increases, decreases or stays the same depending on the geometrical arrangement of the particles.

\subsection{Discussion}

The aim of this work is to better understand the particle-particle interactions appearing in an H-MRE. Several simplifications have been made to set up a first model like the assumption of plane magnetic and mechanical quantities. The 2D model does not represent the case of spherical particles embedded in a matrix, it represents a transversely anisotropic composite containing magnetic cylinders. Thus macroscopic results like the reaction forces are quantitatively not significant but the tendencies can be evaluated as the assumptions are defensible for the local behavior. A comparison to a 3D model has to be done in the future nevertheless. Two other points which have been neglected in this model are the time dependent behavior of the matrix as well as a potential detachment of the particles from the matrix. These aspects have to be included in a future model. Further studies on the influence of an external magnetic field are planned. For this purpose, the assumption of the particles' linear magnetic behavior may no longer be valid. Sugges- tions for modeling the hysteretic behavior of H-MRE have been made for example by Kalina et al. (2017). The case of particles randomly distributed in a matrix is mentioned in the third section of this article. In future works, the presented model will be applied to study the influence of particle distribution and form in representative volume elements (RVE) with periodic boundary conditions.

\section{REFERENCES}

Borcea, L. \& O. Bruno (2001). On the magneto-elastic properties of elastomer-ferromagnet composites. $J$. Mech Phys. Solids 49, 2877-2919.

Cantera, M. A., M. Behrdooz, R. F. Gibson, \& F. Gordaninejad (2017). Modeling of magneto-mechanical response of magnetorheological elastomers (mre) and mre-based systems: a review. Smart Mater. Struct. 26, 023001.

COMSOL (2019). Ac/dc module, forces in stationary fields. COMSOL Documentation 1998-2017.

Danas, K., S. V. Kankanala, \& N. Triantafyllidis (2016). Experiments and modeling of iron-particle-filled magnetorheological elastomers. J. Mech Phys. Solids 60, $120-138$.

Eringen, A. C. \& G. A. Maugin (1990). Electrodynamics of continua 1. Springer-Verlag New York Inc.

Ginder, J., M. Nichols, L. Elie, \& J. Tardiff (1999). Magnetorheological elastomers: properties and applications. Proceedings of the SPIE, Smart Materials Technologies 3675, 131-138.

Han, Y., W. Hong, \& L. E. Faidley (2013). Field-stiffening effect of magneto-rheological elastomers. Int. J. Solids Struct 50, 2281-2919.

Jolly, M. J., J. D. Carlson, \& B. C. Muňoz (1996). A model of the behavior of manetorheological materials. Smart Mater. Struct 5, 607-614.

Kalina, K. A., J. Brummund, P. Metsch, M. Kästner, D. Y. Borin, J. M. Linke, \& S. Odenbach (2017). Modeling of magnetic hysteresis in soft mres filled with ndfeb particles. Smart Mater. Struct. 26, 105019.

Neorem Magnets OY (2019). Characteristic physical properties of sintered ndfeb magnet material at $20^{\circ} \mathrm{C}$. www.neorem.fi, accessed 2019-03-04.

Rigbi, Z. \& L. Jilkén (1983). The response of an elastomer filled with soft ferrite to mechanical and magnetic influences. J. Magn. Magn.Mater. 37, 267-276. 